\title{
Study of the reversing mechanism of wetting agent adding device based on Adams simulation
}

\author{
Guijun $\mathrm{Gao}^{1,2, *}$, and Qiang Dong ${ }^{1,2}$ \\ ${ }^{1}$ Taiyuan University of Technology, College of Mechanical Engineering, Taiyuan, 030024, China \\ ${ }^{2}$ National-Local Joint Engineering Laboratory of Mining Fluid Control, Taiyuan, 030024, China
}

\begin{abstract}
This paper proposed a hydraulically-driven adjustable concentration of wetting agent adding device applied in underground of coal mine. A 3D model of the reversing mechanism of adding device was built by using Solidworks, and then imported to the Adams to build a virtual prototype model. The reversing mechanism was optimized by setting the model parameters, adding constraints and drive. The dynamic characteristics of the reversing mechanism were analysed under different length $l$ of the driven rocker arm. The results show that the value of $l$ $(l=17 \mathrm{~mm})$ can realize automatic reversing. The simulation results provide a basis for trial-manufacture of physical prototyping and optimized design.
\end{abstract}

\section{Introduction}

Dust is the focus of prevention and control in coal industry [1]. Hydraulic dedusting is one of the most simple and effective dedusting methods that can be promoted easily. However, due to the high surface tension of water, fine dust cannot be quickly and effectively wetted by water, and the water's dust-settling efficiency is limited. To improve the ability of wetting coal dust and improve dust-settling efficiency, chemical wetting agents have been used since the 1960s, dedusting with wetting agents has been proven to be an effective dedusting method and is particularly suitable for hydrophobic respiratory dust. The wettability of dust has been studied, J. Liu [2] et al.' study results showed that the increased content of oxygenated groups in high rank coal samples led to decreases in contact angles. Zhenbao Zhao [3] et al.' study results showed that the fixed carbon content of coal dust affects its wettability. Chaohang Xu et al.' [4] study results showed that the wetting time of coal dust is negatively correlated to the moisture content.

The wetting agent needs to be mixed with water to form a solution when it is used, and then the solution is sent to the dedusting equipment through the pipeline. There are some disadvantages in the traditional way of wetting agent adding, for example, the electric driving is required in the quantitative pump adding, it is dangerous in coal mine, the water injection adding method has higher requirements on the sizes of each structure, the reliability is poor, and it is difficult to meet the requirements of the proportion of the wetting agent adding [5-7]. The novel device is shown in Fig. 1, which uses the static pressure water of the coal mine as driving force to pump the high concentration wetting agent liquid directly into the pipeline water. The cylinder body of adding device is

*Corresponding author: Gaogj161@163.com 
equipped with a piston body, both ends of the piston body are equipped with rubber sealing rings, which can make piston slide along the inner wall of the cylinder with different diameters, there is a special structure of the check valve in the piston body, the position of the check valve spool determines the up and down movement of the piston body, the movement of the piston body simultaneously drives the injection piston to move in the injection chamber, after inhalation of the wetting agent, the wetting agent is injected into the adding device. By using the spring rocker arm reversing mechanism, the position of the spool can be controlled so that the piston body is automatically reversed at the extreme position and the injection cycle caused by reciprocating is completed, injection ratio adjustment can be achieved by adjusting the proportional adjusting cylinder.

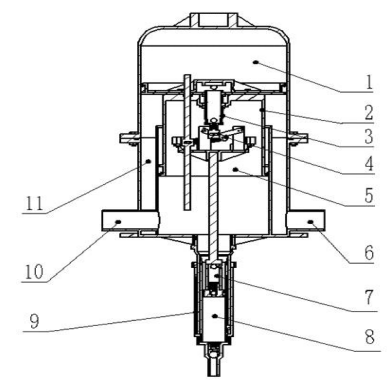

Fig. 1. The schematic diagram of the adding device for wetting agent.

1-the upper chamber, 2-piston body, 3-check valve spool, 4-reversing mechanism, 5-inner chamber, 6-liquid outlet, 7-injection piston, 8-injection chamber, 9- proportional adjusting cylinder, 10-water inlet, 11-outer chamber.

Reversing mechanism is the core of this device. The rationality of movement of the reversing mechanism determines whether the wetting agent adding device can work normally. In this paper, dynamic simulation research on the reversing mechanism is performed by using Adams to determine the key structure size of the reversing mechanism and verify the rationality of the reversing mechanism design.

\section{Structure and working principle}

Fig. 2 shows the structure of the reversing mechanism. The shape of check valve spool that controls the piston body for reciprocating is tubular with two ribs on the top, liquid can flow in from one end with supporting ribs, it can also flow out through the space between the two ribs. Spool maintains good sealing by matching with valve body size. During the switching process of the spool, the direction of the switch is not affected by the pressure of the fluid, and there is only a small sliding frictional resistance on the side surface. The actuator that pushes the check valve movement is constructed by a pair of rocker arms, the two rocker arms interact with two tension springs to create two stable transient positions_ - the upper or the lower position, which can achieve the switch of check valve. Two rockers, active rocker arm and driven rocker arm, can swing up and down around their respective shaft.

In order to explain the working principle of the reversing mechanism, assuming the two rocker arms, spool and the contact rod are at the lower position, the inner chamber communicates with the upper chamber, and the upper and the outer chambers are closed, pressure water flows into the upper chamber through the inner chamber to push down the piston body to drain the water that is in the outer chamber. When the lower end of the contact rod touches the cylinder bottom, the contact rod will be pressed up, it will pluck up the active rocker arm. When the active rocker arm swings over the critical position, the 
spool is quickly pulled to the upper position by the driven rocker arm. The inner and the upper chambers are closed, and the upper chamber communicates with the outer chamber, pressure water flows into the inner chamber to push up the piston body to drain the water in the upper chamber from the outer chamber. Similarly, when the contact rod upper end touches the cylinder top, the contact rod will be pressed down, it will pluck down the active rocker arm, when the active rocker swings over a critical position, the spool is rapidly pulled to the lower position by the driven rocker arm, the piston body moves down. In this way, the piston body continuously reciprocates to complete the injection of the wetting agent.

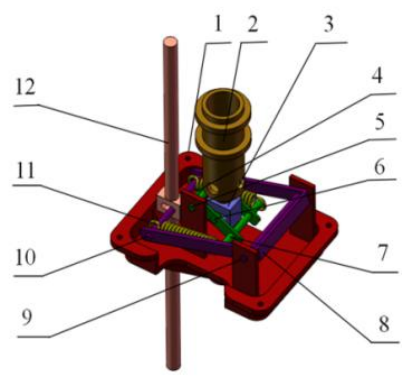

Fig. 2. The structure schematic diagram of the reversing mechanism.

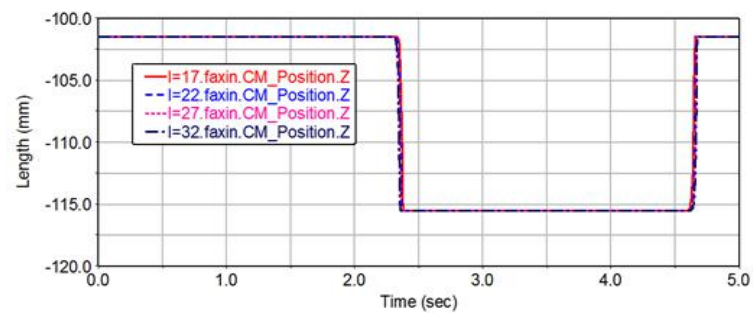

Fig. 3. The displacement curves of the spool at $\mathrm{Z}$ axis.

1-base, 2-spool, 3-linkage, 4-driven rocker arm, 5- driven rocker arm shaft, 6-axis-1, 7-axis-2, 8 -active rocker arm, 9-active rocker arm shaft, 10-axis-3, 11-pulling spring, 12-contact rod.

\section{Modelling and simulation}

Actually, the spool is subjected to external forces such as hydraulic pressure, hydraulic force, and friction in motion, taking the complexity of the forces of the actual mechanism and the balance of main hydraulic pressure acting on the components of the reversing mechanism into account, this paper has ignored the effect of the liquid on the reversing mechanism and make a preliminary discussion of the dynamic characteristics of the reversing mechanism.

The 3D model was made by using Solidworks, the built model was imported into Adams for simulation. The model was simplified without affecting the simulation to reduce computation of solver. some connectors were removed, such as screws between the linkage and the spool, and the base was simplified to a flat plate, the cylinder bottom and top were simplified to two flat plates that is parallel to the base, and the upper and lower valve seats were separated separately.

Material properties were set to stainless steel. The actual reversing mechanism was fixed inside the piston body and moved with it, according to the relativity of motion, the base was fixed to the earth using fixed pairs, the cylinder top and bottom were converted into motion parts by moving pairs, each bearing was simulated with rotating pairs. The relationship between the spool and the upper or lower valve seat, the contact rod and the cylinder top or bottom were simulated by contacting. The Impact Function [8] was used in definition of contact forces. which consisted of two parts: the elastic force generated by cutting in as the mutual contact between the two components occurs and the damping force generated by the relative speed of the two components. The Impact Function is formula (1). 


$$
\text { impact }= \begin{cases}0 & q>q_{0} \\ k\left(q_{0}-q\right)^{e}-c_{\max } \cdot(d q / d t) \cdot \operatorname{step}\left(q, q_{0}-d, 1, q_{0}, 0\right) & q \leq q_{0}\end{cases}
$$

The $q_{0}$ represents initial distance between two objects to be contacted; $q$ represents the actual distance between two objects in the process of contact; $d q / d t$ represents the speed; $k$ represents the stiffness coefficient; $e$ represents the nonlinear index of force, which characterizes the degree of nonlinearity of the material; $c_{\max }$ represents the maximum damping coefficient; $d$ represents the depth of penetration, which characterizes the depth of material cutting in when the damping force reaches its maximum, at the beginning of the contact, the damping force and penetration depth are 0 , with damping force increasing, the penetration depth also gradually increases until the maximum value is reached. In order to avoid the discontinuity of the damping force in the contact process, the Step Function is used, its expression is formula (2).

$$
\begin{array}{cc}
\text { step }=\left\{\begin{array}{lc}
h_{0} & x \leq x_{0} \\
h_{0}+a \cdot \Delta^{2}(3-2 \Delta) & x_{0}<x<x_{1} \\
h_{1} & x \geq x_{1}
\end{array}\right. \\
a=h_{1}-h_{0} \quad \Delta=\left(x-x_{0}\right) /\left(x_{1}-x_{0}\right) &
\end{array}
$$

Referring to the literature [9], the parameters in Adams is shown in table 1.

Table 1. Setting of contact force parameters.

\begin{tabular}{|c|c|}
\hline Contact name & Value \\
\hline Stiffness coefficient $/ \mathrm{N} \cdot \mathrm{mm}^{-1}$ & $1.0 \mathrm{E}+05$ \\
\hline The nonlinear index of force & 1.5 \\
\hline The maximum damping coefficient $/ \mathrm{N} \cdot \mathrm{s} \cdot \mathrm{mm}^{-1}$ & 50 \\
\hline The depth of penetration $/ \mathrm{mm}$ & 0.1 \\
\hline
\end{tabular}

The moving speeds of the cylinder bottom and top are set by using the Step Driving Function, which achieved reciprocating of the cylinder bottom and top within one cycle.

The length of the driven rocker arm was changed by changing the distance $l$ between axis- 1 and axis- 2 on the driven rocker arm. $l=17 \mathrm{~mm}, l=22 \mathrm{~mm}, l=27 \mathrm{~mm}$, and $l=32 \mathrm{~mm}$ were taken respectively to study dynamic characteristics of reversing mechanism. After each change of length, the above model import, model setting and other steps would be repeated.

\section{Comparison of simulation results}

Simulation results under different parameters are compared and analysed. Since the motion of each component is mainly toward Z-axis, this part focuses on the components of each physical quantity in the Z-axis. the length of $l$ in each comparative curve graph is noted.

There is no mutual interference in the reversing mechanism under different values of $l$ in Fig.3, the moving up and down of the spool are realized at the first and second displacement change of spool respectively, and the displacement change is $14 \mathrm{~mm}$. The spool moves smoothly and can be reciprocated between the upper and lower valve seats as required. After the reversing of spool, it is always in close contact with the valve seat and there is no vibration. The performance of the reversing mechanism initially meets the requirements. 


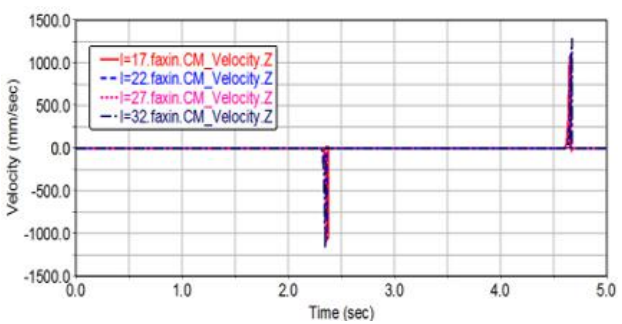

(a)

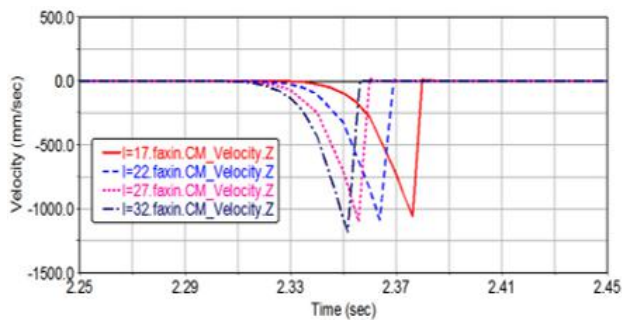

(b)

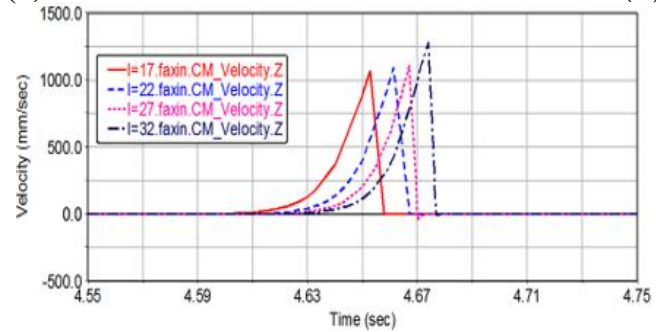

(c)

Fig. 4. The velocity curves of the spool at $Z$ axis.

Fig. 4(a) shows the speed changes of the spool during the entire motion cycle, Fig. 4(b) and 4(c) shows the velocity contrast curve during time of the spool moving up and down respectively. Taking $l=27 \mathrm{~mm}$, the spool starts to move up to reverse at $2.32 \mathrm{~s}$ and its speed reaches maximum of $1010 \mathrm{~mm} / \mathrm{s}$ at $2.36 \mathrm{~s}$, at this time the spool contacts with the upper valve seat, the reversing is completed, the spool starts to move down to reverse at $4.62 \mathrm{~s}$, and its speed reaches maximum of $1103 \mathrm{~mm} / \mathrm{s}$ at $4.66 \mathrm{~s}$, at this time the spool contacts with the lower valve seat, the reversing is completed. It can be seen that the spool reacts sensitively and the reversing time is about $0.04 \mathrm{~s}$, when $l$ increases, the maximum speed achieved by the spool reversing also gradually increases.

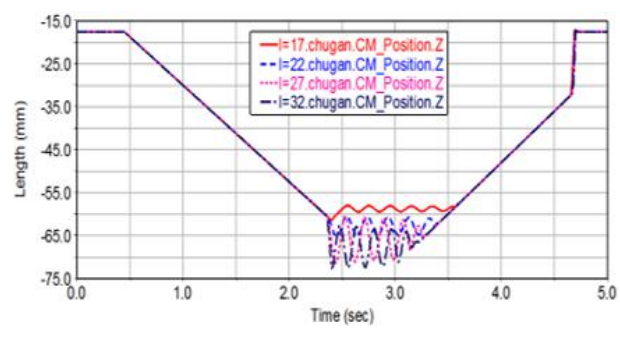

Fig. 5. The displacement curves of the rod at $Z$ axis.

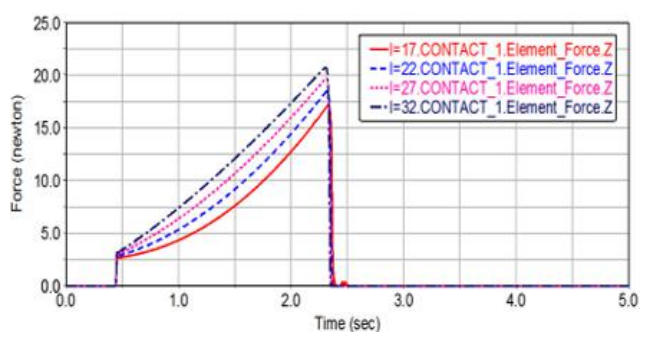

Fig. 6. The curves of the contact force between the rod and cylinder bottom at $\mathrm{z}$ axis.

Fig. 5 shows the displacement curves of the contact rod, taking $l=27 \mathrm{~mm}$, the contact rod starts to move up under the push of the cylinder bottom at $0.44 \mathrm{~s}$, the stroke of the contact rod is approximately $43 \mathrm{~mm}$ when they are in contact with each other, that is $d_{1}$. When reversing occurs at $2.32 \mathrm{~s}$, the contact rod disengages from the cylinder bottom, the displacement changes greatly, then the contact rod waves up and down under the influence of gravity and spring force, the larger the value of $l$ is, the greater the amplitude of the contact rod becomes. The contact rod starts to move down under the push of cylinder top at $3.31 \mathrm{~s}$, the stroke of contact rod is about $32 \mathrm{~mm}$ during the time when they are in contact with each other, that is $d_{2}$. When the reversing occurs at $4.62 \mathrm{~s}$, the contact rod disengages from the cylinder top, and the displacement changes greatly, afterwards the contact rod stops moving under the limit of axis-3 on the active rocker arm. Due to the short reversing 
time of the spool, the displacement of piston body can be considered to 0 during this time. when calculating the stroke $L$ of the piston body, $l_{g t}$ represents the length of cylinder body, $l_{c g}$ represents the length of contact rod. $L=l_{g t} l_{c g}+d_{1}+d_{2}$.

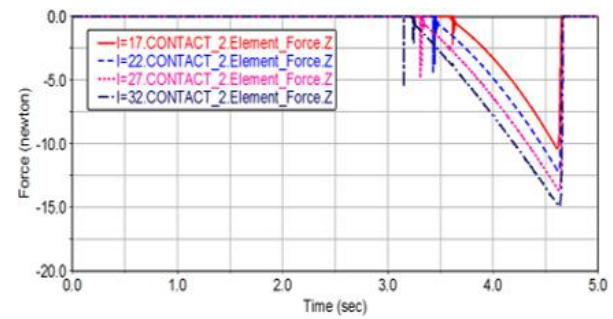

Fig. 7. The curves of the contact force between the rod and cylinder top at $\mathrm{Z}$ axis.

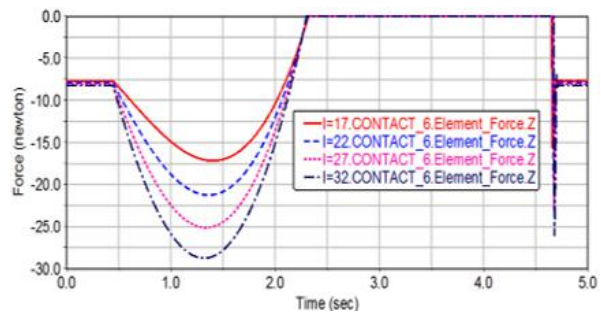

Fig. 8. The curves of the contact force between the spool and lower valve seat at $\mathrm{z}$ axis.

Fig. 6 and Fig. 7 reflect the contact force between contact rod and cylinder bottom, cylinder top respectively, taking $l=27 \mathrm{~mm}$, with the relative motion of the cylinder bottom and the contact rod, the contact force appears at $0.44 \mathrm{~s}$, as the cylinder bottom pushes the contact rod up, the spring deformation increases and the pulling force increases, therefore the contact force between the contact rod and the cylinder bottom gradually increases, when the contact rod disengages from the cylinder bottom at $2.32 \mathrm{~s}$, the spool starts to move up to reverse. Due to the shake of the contact rod, there is contact with the cylinder bottom after it is disengaged from the cylinder bottom, and the force fluctuation occurs. The spool begins to touch the cylinder top at $3.31 \mathrm{~s}$, as the cylinder top pushes the contact rod down, the pulling force of the spring increases due to the deformation increases, and the contact force gradually increases, when the contact rod is out of contact with the cylinder top at $4.62 \mathrm{~s}$, the spool starts to move down to reverse. It can be seen that the longer the $l$ is, the greater the change becomes in the spring deformation, so the larger the contact force is. Due to the gravity effect of the contact rod and other components, the peak of contact force between the contact rod and the cylinder top is smaller than that between contact rod and cylinder bottom. Since the reversing mechanism is driven by the hydraulic pressure, when the liquid pressure is greater than the contact force between the contact rod and the cylinder body, the piston body can continue to maintain the smooth reversing along the original moving direction, it can be seen that although the contact force increases with the increase of $l$, the piston body can complete the reversing smoothly compared with the small driving force of the piston.

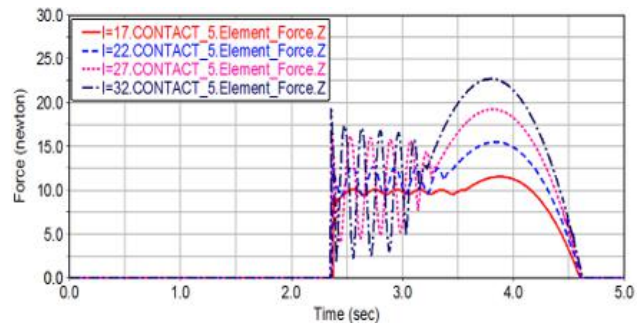

Fig. 9. The curves of the contact force between the spool and upper valve seat at $\mathrm{Z}$ axis.

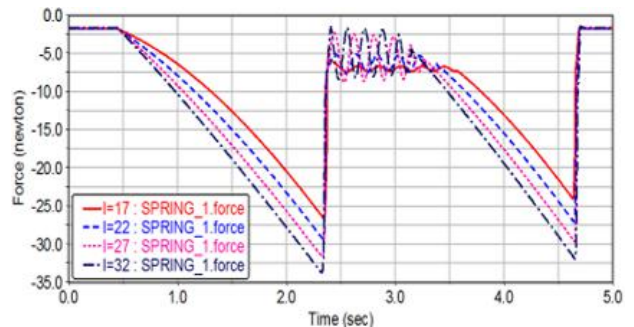

Fig. 10. The curves of the spring force.

Fig. 8 and Fig. 9 show the curves of contact force between the spool and the lower valve seat, the upper valve seat respectively. It can be seen that the spool maintains a certain pressing force with the lower valve seat at the initial time, as the cylinder bottom pushes the contact rod, the moment of pulling force of spring on driven rocker arm changes gradually, it shows that the contact force between the spool and the lower valve seat increases first and 
then decreases, when the spool starts to move up to reverse, the contact force on the lower seat becomes 0 , after reversing, collision force appears on the upper valve seat, the contact force between the spool and the upper valve seat fluctuates due to the spool is affected by the contact rod vibration, the longer the $l$ is, the larger the fluctuation of force becomes. Afterwards, as the cylinder top pushes the contact rod, the contact force between the spool and the upper valve seat first increases and then decreases as the moment of spring pulling force on the driven rocker arm gradually changes, when the spool begins to move down to reverse, the contact force on the upper seat becomes 0 , and collision force appears on the lower seat. Due to the gravity of each component, the contact force that is after the collision of the spool with the lower valve seat quickly remains stable.

Due to the symmetrical distribution of the two springs, the forces are same, taking the one spring for analysis, as shown in Fig. 10, the pulling force of spring is small at the initial time, and the pulling force gradually increases in the process that is from contact to separation between the contact rod and the cylinder bottom, in the reversing process, as the two rocker arms oscillate rapidly, the length of spring shortens and the pulling force decreases rapidly, after the completion of reversing, the pulling force of the spring fluctuates caused by the vibration of contact rod. And the longer the $l$ is, the larger the fluctuation of force becomes. Similarly, the pulling force of spring increases gradually in the process that is from contact between contact rod and cylinder top to the reversing of spool moving down, during the reversing process, the pulling force decreases rapidly, after completion of reversing, the pulling force of spring remains stable due to the gravity force of the contact rod, spool and other components, and the value of spring pulling force returns to the value of initial 0 time.

As the analysis shows, the value of $l(l=17 \mathrm{~mm})$ can be used to complete the reversing. And the mechanical components are less stressed and the force is stable after the reversing.

\section{Conclusion}

(1) Novel adjustable proportion wetting agent adding device applied in underground coal mine is proposed, the device does not need to be driven by electricity and the wetting agent can be added automatically, and the adding concentration is stable and can be adjusted easily.

(2) 3D model of the reversing mechanism of the wetting agent adding device is established, dynamic simulation is performed by changing the different structural parameters of the model, the results show that a smaller value of $l(l=17 \mathrm{~mm})$ can achieve automatic reversing of the reversing mechanism. This device can be applied to agriculture after further optimization.

\section{References}

1. Maoyu Zhang, Fuyin Jia Situation and trend on dust monitoring \& control technologies in underground mine (2011) Journal of Safety Science and Technology 7(12) 188-191

2. J. Liu, M. Holuszko, M. Mastalerz Application of micro-FTIR technique in studying hydrophobicity of coal (2017) International Journal of Coal Geology 178 74-83

3. Zhenbao Zhao, Chen Yang, Chunyan Sun, Xinqian Shu Experimental study of coal dust (2011) Journal of China Coal Society 36(3)

4. Chaohang $\mathrm{Xu}$, Deming Wang et al Effects of chemical properties of coal dust on its wettability (2017) Powder Technology 318 33-39 
5. Yan Kuang Wetting agent selection and adding device (1988) Industrial Safety and Environmental Protection 06 28-30

6. Zhongan Jiang (1999) Wet dust removal technology and its application Beijing. China Coal Industry Publishing House p 135-137

7. Longzhe Jin (1993) The prevention and treatment of mine dust Beijing. China Coal Industry Publishing House p 209-211

8. Zenggang Li (2014) The primary introduction and examples of Adams. National Defend Industry Press pp 126

9. Zuiwei Xie and Xinyue Wu (2007) Analysis of collision Simulation Based on Adams. The 3rd China CAE Analysis Technology Annual Conference 2007 National Symposium for the Advanced CAE Technology \& Application p 347-350 